\title{
PREVALENCIA DE ASPERGILOSIS BRONCOPULMONAR ALÉRGICA EN NIÑOS CON FIBROSIS QUÍSTICA \author{
FIBROSIS
} \\ PREVALENCE OF BRONCHOPULMONARY ASPERGILLOSIS IN CHILDREN WITH CYSTIC
}

\author{
Dra. Catherine Guzmán ', Dra. Genoveva Parra ${ }^{2}$, Dr. Rodrigo Bozzo ${ }^{2}$, EU María José Pinochet ${ }^{2}$, Dra. Marcela Moreno ${ }^{2}$, Dra. Fernanda Cofré ${ }^{2}$, Klgo. Jose Luis \\ Valenzuela ${ }^{2}$, Klgo. Rodrigo Jorquera ${ }^{2}$, Dra. María Angélica Palomino ${ }^{2,3}$ \\ 1.- Becada de Enfermedades Respiratorias Infantiles. Universidad de Chile. \\ 2.- Equipo de Fibrosis Quística. Hospital Roberto del Río. Santiago. \\ 3.- Departamento de Pediatría y Cirugía Infantil Campus Norte. Universidad de Chile y Clínica Las Condes.
}

Aprobado por Comité de Ética del Servicio de Salud Metropolitano Norte.

\section{INTRODUCCIÓN}

La fibrosis quística (FQ) es una enfermedad respiratoria crónica progresiva, de origen genético autosómica recesiva; se estima una incidencia de 1 en 2.500 recién nacidos caucásicos y $1 / 8000$ a $1 / 10000$ recién nacidos en Chile, con aparición de 30 casos nuevos al año (1). La aspergilosis broncopulmonar alérgica (ABPA) es un desorden inmunológico que afecta al pulmón, secundario a una compleja reacción de hipersensibilidad tipo Th2 debido a la colonización bronquial del Aspergillus fumigatus (Af) (2). La ABPA es una complicación importante, pero poco frecuente en niños con fibrosis quística (FQ), cuya prevalencia estimada en promedio es del $10 \%$ según las series publicadas a nivel internacional $(3,4)$. En Chile se desconoce la prevalencia en niños. Entre los factores de riesgo descritos para ABPA en niños con FQ destacan la presencia de atopía y eosinofilia, la terapia prolongada con azitromicina, la colonización crónica por Af, la colonización por stenotrophomona maltophilia y las altas dosis acumulativas de corticosteroides inhalados $(5,6)$. Para el diagnóstico se han establecidos criterios inicialmente descritos por Rosenberg-Patterson en 1978, modificado por Greenberger y recientemente por la Sociedad Internacional de Micología Humana y Animal (ISHAM) $(7,8,9)$. A pesar de ello, aún existe un subdiagnóstico o diagnóstico tardío debido a que la ABPA comparte características clínicas con las exacerbaciones pulmonares de la FQ. En relación al tratamiento, la clasificación de Petterson define la evolución de la enfermedad en 5 estadíos para guiar la terapia, siendo la primera línea los corticosteroides sistémicos para controlar la respuesta inmune del huésped, asociado a agentes antifúngicos que disminuyen la carga del Af y las recurrencias $(10,11)$. Aún existe controversia sobre cuál es la forma óptima de administrar esta terapia, ya

\section{RESUMEN}

La aspergilosis broncopulmonar alérgica (ABPA) es una reacción de hipersensibilidad secundaria al Aspergillus fumigatus (Af) que complica la evolución en fibrosis quística (FQ). Existen pocos estudios pediátricos de su prevalencia publicados en el mundo y en Chile se desconoce. El objetivo de este trabajo fue estimar la prevalencia de ABPA en niños con FQ en un hospital de referencia, explorar factores de riesgo y describir los criterios diagnósticos, tratamiento y evolución. Se incluyeron retrospectivamente los niños con $\mathrm{FQ}$ atendidos en un hospital terciario en Santiago de Chile (Hospital Roberto del Río) entre los años 2011 a 2019, se identificaron aquellos con diagnóstico de ABPA. Se registraron criterios diagnósticos según la Cystic Fibrosis Foundation, presencia de factores de riesgo, tratamientos recibidos y efectos adversos. De 65 pacientes con FQ atendidos en este período, la prevalencia de ABPA fue del $12 \%$. El promedio de edad al diagnóstico fue \pm 11 años (5-17 años), predominando la edad adolescente y el género masculino. El $50 \%$ cumplieron con los criterios clásicos, el $87,5 \%$ usaron antibióticos y el $62,5 \%$ corticoides inhalados. La respuesta favorable al tratamiento inicial con corticoides y antifúngico vía oral fue $62,5 \%$, con una exacerbación al momento del estudio. El $25 \%$ se comportaron como refractario y el $12,5 \%$ respondieron a tratamiento con pulsos de metilprednisolona. El 37,5\% presentaron eventos adversos relacionados a corticoides. La prevalencia de ABPA observada es comparable a las series publicadas. Se necesitan trabajos prospectivos para conocer la prevalencia nacional y su tendencia a lo largo de los años, identificando factores de riesgo.

Palabras claves: Aspergilosis broncopulmonar alérgica, fibrosis quística, prevalencia, Aspergillus fumigatus.

\section{ABSTRACT}

Allergic bronchopulmonary aspergillosis (ABPA) is a hypersensitivity response to Aspergillus fumigatus (Af) and worsens outcome in children with cystic fibrosis (CF). Its prevalence varies in the literature, but we do not know it in Chile. The aim of the study was to know the prevalence of ABPA in children with CF and to describe risk factors, diagnostic criteria, treatment and outcome. We included all patients with CF seen in a tertiary hospital in Santiago, Chile (Hospital Roberto del Río), between 2011 and 2019; ABPA cases (CF Foundation diagnostic criteria) were identified for the estimation of the prevalence. Risk factors, diagnostic criteria and treatment were recorded, as proposed by the Cystic Fibrosis Foundation. A total of 65 patients with CF were identified in the study period, with a prevalence of $12 \%$ ( 8 cases). Mean age at diagnosis \pm 11 years (5-17), more frequent in adolescence and male. CF Foundation criteria diagnostic were identified in $50 \%$ of cases, with high frequency of antibiotic use $(87,5 \%)$ and inhaled steroids $(62,5 \%)$. Positive oral steroids and antifungal treatment response was $62,5 \%$. Refractary response was $25 \%$ and $12,5 \%$ needed intravenous metilprednisolone pulses. A $37,5 \%$ of cases presented adverse effects to steroids. Prevalence of ABPA is comparable to literature. A prospective study is needed to identified national prevalence and trends, identifying risks factors.

Keywords: Allergic bronchopulmonary aspergillosis, cystic fibrosis, prevalence, Aspergillus fumigatus.

que faltan estudios potentes centrados en este grupo pediátrico específico. El objetivo de este estudio fue conocer la prevalencia de ABPA en pacientes atendidos en un hospital terciario en

\section{Autor para correspondencia:}

Dra María Angélica Palomino Montenegro mapalomino@uchile.cl 
Santiago de Chile, describir algunos factores de riesgo y mostrar la experiencia analizando las dificultades diagnósticas y terapéuticas.

\section{PACIENTES Y MÉTODO}

Se realizó un estudio de prevalencia de ABPA en los pacientes con FQ controlados en el Hospital Roberto del Río entre los años 2011 a 2019, previa aprobación del comité de ética de investigación del Servicio de Salud Metropolitano Norte y firma de consentimiento/asentimiento informado. Se cuantificó el número de pacientes con $\mathrm{FQ}$ que fueron atendidos en el policlínico multiprofesional y se seleccionaron aquellos con diagnóstico de ABPA, calculando la prevalencia. Se consideró la clasificación de Patterson para evaluar los episodios de ABPA según la etapa de su evolución. ABPA agudo 0 etapa l es el primer episodio. ABPA en remisión o etapa II es la regresión clínica-radiológica y serológica, con disminución del 35\% en los niveles séricos de lgE a las 6 semanas del inicio de los corticoides. La exacerbación o etapa III es la recurrencia de los síntomas de ABPA con aumento en los niveles séricos de lgE luego de 6 meses de suspender los corticoides. ABPA refractario, dependiente de corticosteroides 0 etapa IV, es cuando se requiere de corticosteroides continuos para prevenir la recurrencia de los síntomas de ABPA. La fibrosis o etapa $V$ es la condición final, con enfermedad fibrocavitaria y de mal pronóstico.

Se registraron variables demográficas tales como edad al diagnóstico, género, lugar de residencia, severidad definida por presencia 0 ausencia de insuficiencia pancreática, IMC previo al diagnóstico de ABPA (eutróficos: z score IMC sobre $\mathrm{p50}$ ), presencia de bronquiectasias previo al diagnóstico, colonización crónica bacteriana o fúngica de la vía aérea y respuesta broncodilatadora significativa definida como cambio VEF1 $\geq 12 \%$.

Los criterios diagnósticos considerados fueron los clásicos y mínimos según la Cystic Fibrosis Foundation (CFF). Criterios clásicos: Deterioro clínico agudo o subagudo no atribuible a otra etiología, lgE total en plasma $>1000$ $\mathrm{Ul} / \mathrm{ml}$, prick test con reactividad $\geq 3 \mathrm{~mm}$ a Af 0 $\lg E(+)$ a Af, precipitinas o lgG anti-Af (+), imágenes nuevas en radiografía o TC (tomografía computada) de tórax que persisten pese a tratamiento con antibióticos. Criterios mínimos: deterioro clínico agudo o subagudo no atribuible a otra etiología, lgE total en plasma $>500$ $\mathrm{UI} / \mathrm{ml}$, prick test con reactividad $>3 \mathrm{~mm}$ a Af 0 $\lg E(+)$ a Af y uno de los siguientes: precipitinas o lgG anti-Af (+), imágenes nuevas en radiografía o TC de tórax con imágenes que persisten pese a antibioterapia.
Se buscaron los factores de riesgo para ABPA (presencia de atopia definida como sensibilización a $\geq 2$ aeroalergenos), uso de azitromicina prolongada, colonización crónica por Pseudomona Aeurginosa, uso de corticoides inhalados y colonización por Af.

Se consignó el tratamiento recibido: corticoide oral o endovenoso, duración del tratamiento en meses, criterios de respuesta a tratamiento: mejoría clínica, de función pulmonar (VEF1), descenso de $\lg E \geq 25 \%$ del valor inicial a las 8 semanas de tratamiento y resolución de imágenes radiológicas. Los efectos adversos del tratamiento corticoidal consignados fueron hiperglicemia con o sin requerimiento de insulina, hipertensión arterial, alteraciones psiquiátricas y los efectos adversos gastrointestinales, dermatológicos y hepáticos del tratamiento antifúngico.

\section{RESULTADOS}

Entre los años 2011 y 2019 hemos controlado 65 niños y adolescentes con diagnóstico de FQ en el Hospital Roberto del Río en un policlínico especializado y multiprofesional. En este período, 8 pacientes tuvieron ABPA agudo, 3 evolucionaron con exacerbaciones y un mismo paciente se consideró como refractario a los corticoides orales, pero con buena respuesta a metilprednisolona endovenosa. Todos los pacientes con recurrencia del ABPA tenían algún grado de compromiso nutricional. Considerando los 8 pacientes con ABPA agudo, la prevalencia fue de $12 \%$. La distribución de los casos en el período analizado se muestra en la Fig. 1. La edad de presentación del primer episodio de ABPA osciló entre los 5 y 17 años, todos los casos fueron hombres, el 62,5\% fueron adolescentes y el $37,4 \%$ residían fuera de la Región Metropolitana. Previo al episodio de ABPA, todos presentaban insuficiencia pancreática, un 62,5\% (5/8) tenía IMC $<$ p50, un $62,5 \%(5 / 8)$ tenían bronquiectasias y un $50 \%$ presentaron respuesta broncodilatadora significativa en el examen de función pumonar, todos manejados con corticoides inhalatorios asociado a broncodilatador de acción prolongada. Ningún paciente presentó colonización crónica con Af. El resto de las variables evaluadas junto con los factores de riesgo se describen en la tabla 1. El 91,6\% (11/12) de los episodios de ABPA se presentaron con deterioro clínico: 10 tuvieron aumento de secreciones, tres de ellos de color café oscuro y el 58,3\% presentaron deterioro de la función pulmonar. De acuerdo a los criterios diagnósticos de ABPA agudo, 4 pacientes cumplieron con el diagnóstico según criterios clásicos, 2 casos se consideraron dentro de los criterios mínimos y 2 casos no completaron los criterios, pero respondieron a tratamiento. Ninguno contó con determinación de precipitinas ó lgG para Af. El detalle de los criterios diagnósticos para cada caso se presentan en la tabla 2. En el manejo de los 8 casos de ABPA agudo, 7 recibieron tratamiento ambulatorio completo con prednisona asociado a antifúngico oral (itraconazol); un paciente recibió prednisona por 2 meses, pero debido a mala adherencia continuó con pulsos de metilprednisolona endovenosa. El promedio de tratamiento con corticoides orales fue de 18 semanas ( 16 - 24 semanas) asociado a antifúngico por 2 a 5 meses. La respuesta favorable, considerando mejoría clínica y el descenso de la lgE, se observó en 4 de los 7 casos tratados con prednisona y un caso fue refractario. En 2 de ellos no se obtuvo IgE de control, sin embargo no han presentado nuevas exacerbaciones al momento del estudio.

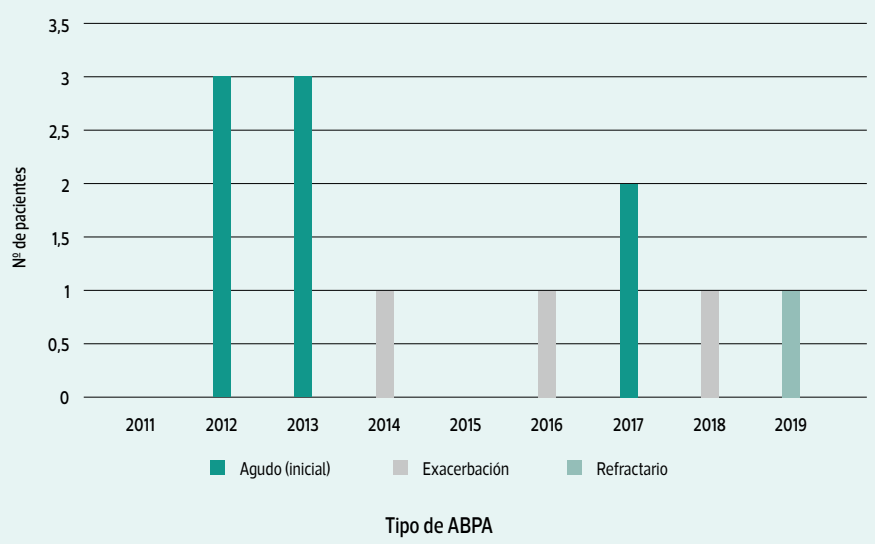

Figura 1. Distribución de los episodios de aspergilosis broncopulmonar alérgica en los pacientes con FQ atendidos en el policlínico del Hospital Roberto del Río entre los años 2011 a 2019, Santiago, Chile. 
Tabla 1. Caracterización de los pacientes con aspergilosis broncopulmonar alérgica controlados en el Hospital Roberto del Río entre los años 2011-2019, Santiago, Chile.

\begin{tabular}{|c|c|c|c|c|c|c|c|}
\hline Caso & $\begin{array}{c}\text { Edad al } \\
\text { disgnóstico de } \\
\text { ABPA (años) }\end{array}$ & $\begin{array}{c}\text { IMCZ } \\
\text { score }\end{array}$ & $\begin{array}{c}\text { VEF1 } \geq 12 \% \\
\text { (Post-B2) }\end{array}$ & $\begin{array}{c}\text { Colonización } \\
\text { crónica }\end{array}$ & $\begin{array}{c}\text { CI } \\
\text { crónico }\end{array}$ & $\begin{array}{c}\text { Azitromicina } \\
\text { crónica }\end{array}$ & $\begin{array}{c}\text { AT } \\
\text { inhalado }\end{array}$ \\
\hline 1 & 13 & -0.28 & + & PA & + & + & + \\
\hline 2 & 12 & +0.07 & - & SAMS & - & + & + \\
\hline 3 & 17 & -1.86 & - & - & - & + & + \\
\hline 4 & 9 & +1.37 & - & SAMS & - & + & - \\
\hline 5 & 5 & -1.40 & + & - & + & - & - \\
\hline 6 & 11 & -1.34 & + & SAMS & + & + & - \\
\hline 7 & 7 & -0.17 & + & - & + & + & + \\
\hline 8 & 17 & +0.04 & + & - & + & + & + \\
\hline
\end{tabular}

ABPA: Aspergilosis broncopulmonar alérgica, IMC: índice de masa corporal, PA: Pseudomona aeruginosa, SAMS: Staphylococcus aureus meticilino sensible, Cl: corticoide inhalado, ATB: antibiótico

Tabla 2. Criterios diagnósticos de aspergilosis broncopulmonar alérgica según la Cystic Fibrosis Foundation.

\begin{tabular}{|c|c|c|c|c|c|c|}
\hline Caso & $\begin{array}{c}\text { Deterioro } \\
\text { Clínico }\end{array}$ & $\begin{array}{c}\% \text { caída } \\
\text { VEF1 }\end{array}$ & $\begin{array}{c}\mathrm{IgE}>1000 \\
\mathrm{UI} / \mathrm{mL}\end{array}$ & $\begin{array}{l}\text { Prick test } \\
\text { Af } / \operatorname{lgE} A F\end{array}$ & $\begin{array}{c}\text { Precipitinas/ } \\
\text { IgG } A F\end{array}$ & $\begin{array}{l}\text { Rx o TC de } \\
\text { tórax alterada }\end{array}$ \\
\hline 1 & $\begin{array}{c}\text { Aumento } \\
\text { secreciones }\end{array}$ & 21 & 1540 & $+1+$ & $\mathrm{NE}$ & - \\
\hline 2 & $\begin{array}{c}\text { Aumento } \\
\text { secreciones }\end{array}$ & 26 & 33 & $+1+$ & $\mathrm{NE}$ & $\mathrm{NE}$ \\
\hline 3 & Sibilancias & 10 & 633 & $+/ \mathrm{NE}$ & $\mathrm{NE}$ & + \\
\hline 4 & $\begin{array}{l}\text { Aumento } \\
\text { secreciones }\end{array}$ & 0 & 1128 & $\mathrm{SR} / \mathrm{NE}$ & $\mathrm{NE}$ & + \\
\hline 5 & Asintomático & 0 & 1460 & $+/ N E$ & $\mathrm{NE}$ & - \\
\hline 6 & $\begin{array}{l}\text { Aumento } \\
\text { secreciones }\end{array}$ & 10 & 1975 & $+/ N E$ & $\mathrm{NE}$ & + \\
\hline 7 & $\begin{array}{l}\text { Aumento } \\
\text { secreciones }\end{array}$ & 8 & 701 & $+1-$ & $\mathrm{NE}$ & NE \\
\hline 8 & $\begin{array}{l}\text { Aumento } \\
\text { secreciones }\end{array}$ & 2 & $\mathrm{NE}$ & $+1+$ & $\mathrm{NE}$ & $\mathrm{NE}$ \\
\hline
\end{tabular}

ABPA: aspergilosis broncopulmonar alérgica, CFF: Cystic Fibrosis Foundation, IgE: inmunoglobulina E, Af: Aspergillus fumigatus, SR: sin reactivo disponible lgG: inmunoglubulina G, Rx: radiografía, TC: tomografía computada, NE: no evaluado.

En todos los casos de exacerbación, incluído el refractario, el tratamiento incluyó 4 a 5 pulsos de metilprednisolona mensuales asociados a voriconazol por 2 meses. Los efectos adversos observados fueron la hiperglicemia en relación a los pulsos de metilprednisolona en 2 niños con diagnóstico previo de diabetes mellitus tipo 1 y en ellos fue necesario aumentar las dosis de insulina de manera transitoria; en 1 adolescente presento hipertensión arterial asociado al uso prolongado de prednisona. Un niño con antecedentes de terrores nocturnos y que recibió prednisona presentó síntomas psiquiátricos caracterizados por alucinaciones. No hubo efectos adversos a los antifúngicos.

\section{DISCUSIÓN}

ABPA es una reacción de hipersensibilidad compleja frente al Af y que complica el curso clínico de los pacientes con FQ (10). En nuestro estudio encontramos una prevalencia de ABPA durante el período estudiado de 8 años de un $12 \%$, considerando como caso aquellos con ABPA agudo. En esta casuística la prevalencia obtenida podría ser comparable con las series clínicas publicada en la literatura en pacientes pediátricos. El predominio de casos en la edad adolescente, el género masculino y la ausencia del diagnóstico de ABPA en menores de 5 años en nuestro es- tudio fue similar a lo reportado en las publicaciones $(6,13)$. Existió dificultad para establecer el diagnóstico, principalmente por la sobreposición de síntomas y hallazgos radiológicos de una exacerbación infecciosa y ABPA y la falta de uniformidad en los criterios utilizados. Este problema se repite en la literatura (12). En nuestra serie, 4 pacientes cumplieron con criterios clásicos, uno con criterios mínimos, y en los 2 casos que no lograron completar los criterios mínimos, prevaleció la alta sospecha clínica junto al deterioro de la función pulmonar, presentando una satisfactoria evolución con el tratamiento. La medición de la lgE sérica, como está establecido en las guías internacionales, permitió sospechar el diagnóstico en 3 casos con limitada expresión clínica, siendo la lgE una herramienta relevante tanto para el diagnóstico como el seguimiento de estos pacientes (14). Entre los factores de riesgo descritos en la literatura para ABPA encontrado en nuestros casos, destacó el compromiso nutricional, uso de antibióticos y corticoides inhalados. De los 8 pacientes que se trataron con prednisona y antifúngicos, 7 tuvieron buena respuesta al tratamiento; esto incluyó a los 3 pacientes con lgE< $500 \mathrm{lU} / \mathrm{ml}$. La respuesta favorable al tratamiento confirmó el diagnóstico de ABPA en estos casos. Parece necesario complementar el estudio con laboratorio más específico frente a la sospecha clínica de ABPA en un paciente con FQ e lgE < $500 \mathrm{lU} / \mathrm{ml}$ y así iniciar precozmente el tratamiento adecuado. La mayoría de las guías recomiendan el uso de corticosteroides orales, sin embargo, existen reportes de pulsos de metilprednisolona con resultados positivos en la estabilización clínica y la disminución de las exacerbaciones $(15,16)$. En nuestra experiencia el uso de pulsos de metilprednisolona nos permitió tratar la falta de adherencia al tratamiento y manejar mejor complicaciones como la hiperglicemia en pacientes con diabetes asociado a FQ, aumentando las dosis de insulina solo durante los 3 días del uso de corticoides. El esquema utilizado en estos casos fue el propuesto por Cohen (15). Este estudio aporta información sobre las dificultades en el diagnóstico y manejo de esta complicación de la FQ. A pesar de la experiencia clínica, existe limitada evidencia de la epidemiología, patogénesis, criterios diagnósticos, clasificación y tratamientos efectivos (9). La CFF ha definido los criterios diagnósticos de ABPA(14). Frente a la ausencia de los criterios diagnósticos clásicos se requiere un alto índice de sospecha e integrar la información clínica con los hallazgos de laboratorio y radiológicos. La respuesta al tratamiento con corticoides sistémicos, evaluada desde el punto de vista clínico y de laboratorio, confirma en definitiva el diagnóstico de ABPA. 
Con frecuencia existe dificultad para reconocer la enfermedad, lo que lleva a un sub-diagnóstico, existiendo en ocasiones retraso en años desde el inicio de los síntomas y el diagnóstico definitivo (9). Este subdiagnóstico condiciona un manejo tardío con el consecuente deterioro funcional y aumento del daño pulmonar con la aparición y/o extensión de bronquiectasias (5). Si el diagnóstico y tratamiento son oportunos, mejora la calidad de vida, la función pulmonar y se reduce el uso de tratamientos innecesarios como los antibióticos endovenosos. Es por esta razón que los centros especializados en la atención de niños con FQ requieren de un protocolo de diagnóstico y tratamiento, como lo proponen las guías internacionales y nacionales $(14,17)$. Es importante identificar a aquellos pacientes con $\mathrm{FQ}$ que tienen mayor riesgo de sensibilizarse al Af, como la condición de atopía, la presencia de bronquiectasias y la presencia de cultivos positivos para AF. Nuestro estudio tiene la limitación de ser retrospectivo en la recolección de la información de cada caso, acotado al tiempo de 8 años desde que se inicia el manejo multidisciplinario organizado en nuestro hospital. Los criterios de diagnóstico pueden haber variado a lo largo de los años, dificultad también observada en la literatura.

\section{CONCLUSIONES}

La prevalencia de ABPA en nuestro centro es similar a la descrita en otras series de casos, logrando en la mayoría de los casos un diagnóstico de acuerdo con lo propuesto por la CFF. Es importante tener alto índice de sospecha, manteniendo un protocolo de pesquisa de factores de riesgo, diagnóstico y tratamientos más adecuados. Se requiere un estudio prospectivo nacional con un protocolo estandarizado de diagnóstico y tratamiento de acuerdo con lo propuesto en el consenso chileno para el manejo de pacientes con fibrosis quística (17)

\section{de interés.}

Los autores declaran no tener conflictos

\section{Revisores de este artículo \\ Dra. María Lina Boza \\ Profesora Adjunta de Pediatría, \\ Universidad de Chile. \\ Dra. Viviana Lezana \\ Profesora Adjunta, \\ Universidad de Valparaíso.}

Fecha de recepción: enero 2021

Fecha de publicación: junio 2021

\section{REFERÊNCIAS}

1. Orientaciones técnicas para la atención integral de fibrosis quística. Segunda edición (Internet) Chile: Grupo Técnico, Programa de Fibrosis Quística Departamento Procesos Clínicos Integrados Subsecretaría de Redes Asistenciales Ministerio de Salud, Gobierno de Chile; c2019 (citado enero 2021). Disponible en: https://www.minsal.cl/wp-content/uploads/2019/08/ORIENTACIONES-T\%C3\%89CNICAS-FIBROSIS-QUISTICA-2019.pdf

2. Latgé J-P, Chamilos G. Aspergillus fumigatus and aspergillosis in 2019. Clin Microbiol Rev 2019, 33: e00140-18. doi: 10.1128/CMR.00140-18.

3. Janahi IA, Rehman A, Al-Naimi AR. Allergic bronchopulmonary aspergillosis in patients with cystic fibrosis. Ann Thorac Med 2017;12:74-82. doi: 10.4103/atm.ATM_231_16

4. Maturu VN, Agarwal R. Prevalence of Aspergillus sensitization and allergic bronchopulmonary aspergillosis in cystic fibrosis: systematic review and meta-analysis. Clin Exp Allergy. 2015; 45(12):1765-78. doi: 10.1111/cea.12595.

5. Jat KR, Vaidya PC, Mathew JL, Jondhale S, Singh M. Childhood allergic bronchopulmonary aspergillosis. Lung India 2018;35:499-507. doi: 10.4103/lungindia.lungindia_216_18.

6. Sharma VK, Raj D, Xess I, Rodha R, Kabra AK. Prevalence and risk factors for allergic bronchopulmonary aspergillosis in Indian children with cystic fibrosis. Indian Pediatr. 2014; 51(4):295-7.

7. Rosenberg M, Patterson R, Roberts M, Wang J. The assessment immunologic and clinical changes occurring during corticosteroid the therapy for allergic bronchopulmonary aspergillosis. The American Journal of Medicine 1978;64(4):599-606. doi: 10.1016/0002 9343(78) $90579-\mathrm{x}$.

8. Greenberger PA. Allergic bronchopulmonary aspergillosis J Allergy Clin Immunol. 2002;110(5):685-92. doi: 10.1067/ mai.2002.130179.

9. Agarwal R, Chakrabarti A, Shah A, Gupta D, Meis JF, Guleria $\mathrm{R}$, et al. ABPA complicating asthma ISHAM working group. Allergic bronchopulmonary aspergillosis: review of literature and proposal of new diagnostic and classification criteria. Clin Exp Allergy. 2013 Aug;43(8):850-73. doi: 10.1111/ cea.12141. PMID: 23889240

10. Mahdavinia M, Grammer LC. Management of allergic bronchopulmonary aspergillosis: a review and update. Ther Adv Respir Dis. 2012;6(3):173-87. doi 10.1177/1753465812443094

11. Agarwal R, Aggarwal AN, Dhooria S, Singh Sehgal I, Garg M, Saikia B, et al. A randomised trial of glucocorticoids in acute-stage allergic bronchopulmonary aspergillosis complicating asthma. Eur Respir J. 2016;47(2):490-8. doi 10.1183/13993003.01475-2015. Epub 2015 Nov 19. PMID 26585431.

12. Knutsen AP, Slavin RG. Allergic bronchopulmonary aspergillosis in asthma and cystic fibrosis. Clin Dev Immunol. 2011:843763. doi: 10.1155/2011/843763.

13. Armstead J, Morris J, Denning DW. Multi-Country Estimate of Different Manifestations of Aspergillosis in Cystic FibroSis. PLoS ONE 2014; 9(6): e98502.

14. Stevens DA, Moss RB, Kurup VP, Knutsen AP, Greenberger $P$, Judson MA, et al. Allergic bronchopulmonary aspergillosis in cystic fibrosis--state of the art: Cystic Fibrosis Foundation Consensus Conference. Clin Infect Dis. 2003;37 Suppl 3:S225-64. doi: 10.1086/376525. Erratum in: Clin Infect Dis. 2004 Jan 1;38(1):158. PMID: 12975753

15. Cohen-Cymberknoh M, Blau H, Shoseyov D, Mei-Zahav M, Efrati O, Armoni S, et al. Intravenous monthly pulse methylprednisolone treatment for ABPA in patients with cystic fibrosis. J Cyst Fibros. 2009;8(4):253-7. doi: 10.1016/j. jcf.2009.04.008. Epub 2009 May 15. PMID: 19447081.

16. Singh Sehgal I, Agarwal R. Pulse methylprednisolo- ne in allergic bronchopulmonary aspergillosis exacerbations. Eur Respir Rev. 2014 1;23(131):149-52. doi: 10.1183/09059180.00004813.

17. Boza ML, Melo J, Barja S, Ethel Codner D, Patricio Gomolan G, Rubén Hernández M, et al. Consenso chileno para la atención integral de niños y adultos con Fibrosis Quística. Neumol Pediatr. 2020; 15(4): 429-483. DOI https://doi. org/10.51451/np.v15i4.123 